\title{
A RARE INTRATHORACIC TUMOUR
}

\author{
Rahul Zamad, S. Dasgupta, P. B. Nichkaode, Abhay Chaudhary, Nilesh Tulaskar
}

1. Resident. Department of Surgery, NKP Salve Institute of Medical Sciences. Hingna, Nagpur.

2. Professor. Department of Surgery, NKP Salve Institute of Medical Sciences. Hingna, Nagpur.

3. Associate Professor. Department of Surgery, NKP Salve Institute of Medical Sciences. Hingna ,Nagpur.

4. Lecturer. Department of Surgery, NKP Salve Institute of Medical Sciences. Hingna Nagpur.

5. Lecturer. Department of Surgery, NKP Salve Institute of Medical Sciences. Hingna Nagpur.

\section{CORRESPONDING AUTHOR:}

Dr. Rahul C. Zamad,

27, Congress Nagar,

Tapsya Bhavan,

Nagpur- 440012.

E-mail: zamad.rahul@gmail.com

ABSTRACT: The study presents a case of 42-year-old male with complaints of Pain in right hypochondrium since 6 months. Imaging studies were suggestive of a large hypodense mass in area of Rt. lobe of liver with adjacent Rt. pleural effusion \& basal consolidation. Diagnostic laparoscopy performed and biopsy taken which showed Spindle cell variant of malignant mesothelioma. Excision of mass was performed by right posterolateral thoracotomy approach. Histopathology showed highly atypical spindle cell tumour with Immunohistochemistry- S/o low grade leiomyosarcoma. Patient relieved symptomatically and no recurrence at 12 months of follow up.

KEY WORDS: spindle cell tumour; leiomyosarcoma; mesothelioma; thoracotomy

INTRODUCTION: Leiomyosarcoma of the diaphragm is very rare. Primary tumours of the diaphragm are rare lesions. Leiomyosarcoma of the diaphragm is exceptional. The first case has been reported by Kirschbaum in 1935 (1); and so far only ten cases have been reported in the literature to the best of our knowledge (8). Due to the lack of symptoms, and unresponsiveness to various therapeutic modalities, the prognosis of leiomyosarcoma is very poor. Soft tissue sarcomas are considerably rare and stand for only approximately $0.7 \%$ of the malignancies in the entire population.(7) Leiomyosarcomas can be situated in different sites yet above half of cases occur in the organs of the abdomen and retroperitoneal space. They metastasise to the liver and lungs via blood or less common the lymphatic vessels. The treatment of choice in the early stages of sarcoma is surgical operation (5)

CASE REPORT: 42 years old male patient, non-smoker, presented with complaint of pain in right upper abdomen since 6 months. Patient was otherwise asymptomatic. Systemic examination was within normal limits with Air entry decreased on right lung bases. Routine blood investigations were within normal limits. Tumour markers CEA, CA19-9, and AFP were also within normal limits. X-ray Chest was S/o blunting of right Costo-phrenic angle and compressed right lower lobe of lung .Ultrasonography revealed a well defined hypoechoic rounded mass in subdiaphragmatic region in right lobe of liver measuring $83.5 \times 89.8 \mathrm{~mm} \mathrm{~s} / 0$ large mass in liver with right pleural effusion. CECT scan of thorax and upper abdomen was performed which showed a large hypodense mass in postero -superior aspect of right lobe of liver with adjacent right pleural effusion \& basal consolidation? Organized liver abscess??? Mass 
lesion.(fig 1).subsequently pleural fluid cytology was performed which came out to be adenocarcinoma of lung. CT guided biopsy was performed which proved to be Spindle cell tumor S/o smooth muscle tumor, ?Bizarre leiomyoma, ???Leiomyosarcoma. Decision for diagnostic laparoscopy was taken which revealed fleshy mass, just below diaphragm abutting upper border of liver from which Trucut biopsy taken which came out to be Spindle cell variant of malignant mesothelioma

Patient was posted for surgery and right posterolateral thoracotomy was performed. Tumour was seen arising from right hemidiaphragm, (fig 2) Rt. lung and pleura were completely separated from tumor and excised along with $2 \mathrm{~cm}$ margin of diaphragm. It was involving the superior border of liver but was clearly separated from it. Diaphragmatic defect was closed with edge to edge sutures with Prolene no 1 and intermittent polyester. Delivered specimen was a Well encapsulated tumor of size $8 \times 6$ Inches weighing around 500gms. Cut surface showed Fish flesh like appearance with no areas of necrosis $n$ calcification. Post operative recovery was uneventful. Lung expansion was confirmed by X-ray chest on $6^{\text {th }}$ post operative day. Histopathological examination revealed proliferation of highly atypical spindle cells.(fig 3 ) Immunohistochemical studies revealed presence of muscle vimentin, desmin and actin in the ,neoplastic cells and morphology suggested leiomyosarcoma G2 stage. Patient was discharged on post-op day 14 .

DISCUSSION: Leiomyosarcoma develops relatively frequently in the uterus and digestive tract, and somewhat less frequently in the skin, ovary, retroperitoneum and mesentery. On the other hand, primary leiomyosarcoma of the diaphragm is extremely rare. As these tumors are mostly asymptomatic or occasionally present nonspecific symptoms, leiomyosarcomas of the diaphragm often grow large before they are detected. The rarity of this tumor makes its early identification much harder. It is often found on chest radiography first, which reveals, however, non-specific findings such as deformation or elevation of the diaphragmatic shadow. For establishment of diagnosis, US, CT and MRI are useful; In particular, MRI is useful in localizing the tumor and determining the relationship with other organs. The only qualitative diagnostic method is biopsy, although it is often difficult. In the present case, diagnosis was established by biopsy under laparoscopic guidance. In treating leiomyosarcoma, resection is the most promising approach. Both chemotherapy and radiotherapy are less effective. Parker (5) and Strauch (7) reported that a complete cure could be obtained only by surgery in leiomyosarcoma of the diaphragm. Except for the case in a 2-month-old child of Koudriavstev,(4)out of first seven cases reported the treatment and prognosis of the previous seven cases were as follows. Only surgical resection was done in four cases. Two patients died at 9 months and 14 months. Two other patients were still alive at 12 months and 24 months. Only radiotherapy was done in two cases. One died within 3 months and the other in 26 months. In only one case (6) was combination chemotherapy done for relapse after surgery, and the patient was still alive after 51 months. Actually, physical signs are mostly absent for tumor of the diaphragm. Therefore such tumors are diagnosed late and are already large. Once the tumor invades the surrounding tissue, curative operation becomes difficult and the prognosis with only surgical treatment is poor as in the present case. On the other hand, Blondeel (6) treated a patient with combination chemotherapy for relapse after surgery. The chemotherapy consisted of Iphosphamide $(5 \mathrm{~g} / \mathrm{m} 2$ over 3 days) and Adriamycin (50 mg/m2 day 1) repeated every 3 weeks. In total, the patient received six cycles, resulting in very good partial remission and was alive after 51 months. Thus, in treating for leiomyosarcoma of the diaphragm, we should consider not only operation but 
also chemotherapy for relapse after surgery or incomplete resection. We would thus obtain a better prognosis.

\section{REFERENCES}

1. 1.Kirschbaum JD. Myosarcoma of the diaphragm. Ann J Cancer 1935; 25: 730-7.

2. McCoy WC. Leiomyosarcoma of the diaphragm. South Med J 1963; 56: 642-7.

3. Dionne Gp, Beland JE, Nai-San Wang. Primary leiomyosaroma of the diaphragm of an asbestos worker. Arch Pathol Lab Med 1976; 100: 398.

4. Koudriavstev VA, Ponomarieva OA. Malignant tumour of the diaphragm in a 2-monthold child. Vestin Khir 1978; 120: 85-6.

5. Parker MC. Leiomyosarcoma of the diaphragm: a case report. Eur J Surg Oncol 1985; 11: 171-5.

6. Blondeel $\mathrm{Ph} \mathrm{N}$, Christiaens MR, Thomas J, et al. Primary leiomyosarcoma of the diaphragm. Eur J Surg Oncol 1995; 21: 429-31.

7. Strauch JT, Aleksic I, Schorn B, et al. Leiomyosarcoma of the diaphragm. Ann Thorac Surg 1999; 67: 1154-5.

8. Louzi, A.; Benelkhaiat, R.; Finech, B et al Leiomyosarcoma of the diaphragm: two case reports. The Internet Journal of Surgery | December 2, 2007|. 


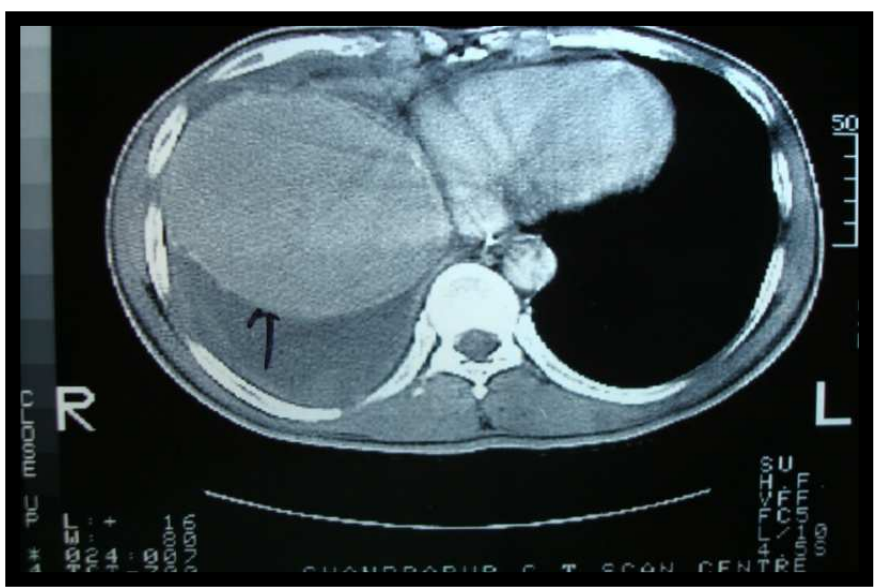

Fig 1 showing large hypodense mass in postero -superior aspect of right lobe of liver with adjacent right pleural effusion \& basal consolidation

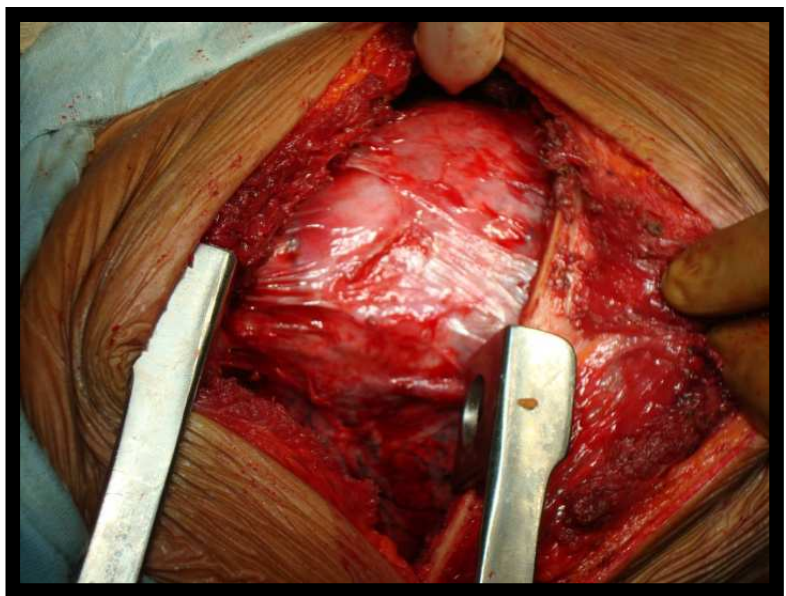

Fig 2 showing tumour arising from right hemidiaphragm

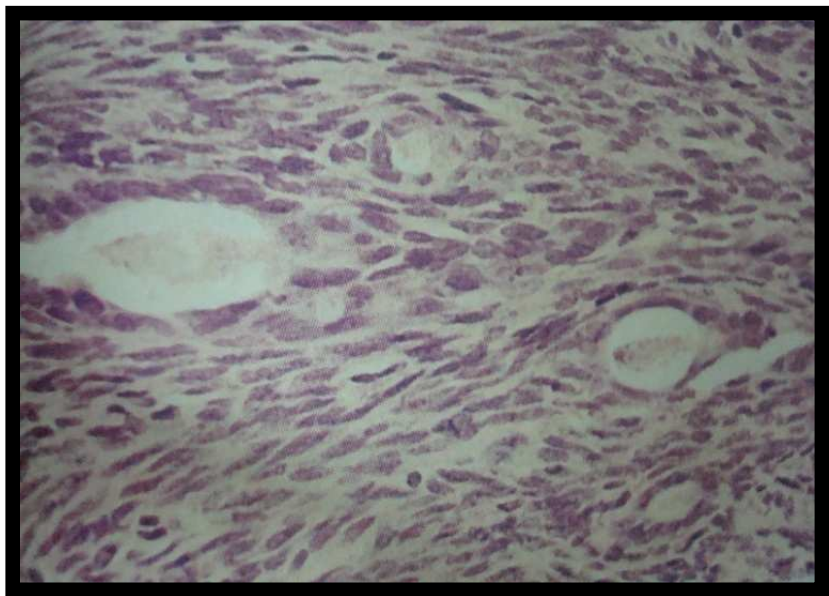

Fig 3 Histopathological specimen showing proliferation of highly atypical spindle cells 